\title{
APPLICATION OF DEEP LEARNING OF MULTI-TEMPORAL SENTINEL-1 IMAGES FOR THE CLASSIFICATION OF COASTAL VEGETATION ZONE OF THE DANUBE DELTA
}

\author{
S. Niculescu ${ }^{1}$, D. Ienco ${ }^{2}$, J. Hanganu ${ }^{3}$ \\ ${ }^{1}$ Université de Bretagne Occidentale, CNRS, LETG Brest UMR 6554 CNRS, France - simona.niculescu@univ-brest.fr \\ ${ }^{2}$ Maison de la Télédétection, IRSTEA - UMR TETIS, Univ. de Montpellier, Montpellier, France \\ ${ }^{3}$ Danube Delta National Institute for Research and Development, Romania
}

\section{Commission III, WG III/7}

KEY WORDS: Deep Learning, vegetation, Danube Delta, temporal series Sentinel-1.

\begin{abstract}
:
Land cover is a fundamental variable for regional planning, as well as for the study and understanding of the environment. This work propose a multi-temporal approach relying on a fusion of radar multi-sensor data and information collected by the latest sensor (Sentinel-1) with a view to obtaining better results than traditional image processing techniques. The Danube Delta is the site for this work. The spatial approach relies on new spatial analysis technologies and methodologies: Deep Learning of multi-temporal Sentinel-1. We propose a deep learning network for image classification which exploits the multi-temporal characteristic of Sentinel1 data. The model we employ is a Gated Recurrent Unit (GRU) Network, a recurrent neural network that explicitly takes into account the time dimension via a gated mechanism to perform the final prediction. The main quality of the GRU network is its ability to consider only the important part of the information coming from the temporal data discarding the irrelevant information via a forgetting mechanism. We propose to use such network structure to classify a series of images Sentinel-1 (20 Sentinel-1 images acquired between 9.10.2014 and 01.04.2016). The results are compared with results of the classification of Random Forest.
\end{abstract}

\section{INTRODUCTION}

Land cover is a fundamental variable for regional planning, as well as for the study and understanding of the environment. This topic has become a key element of most inventory maps and monitoring inventories of environmental phenomena. The active or passive remote sensors used for various applications related to the detection, analysis, mapping and definition of land cover changes and vegetation monitoring cover a very broad domain of the electromagnetic spectrum. Remote-sensing technologies can deliver data on habitat quantity (amount, configuration) and quality (e.g., structure, distribution of individual plant species, habitat types and/or communities, persistence (He et al., 2011) across a range of spatial resolutions and temporal frequencies (Wulder et al., 2004).

The new generation of radar imagery has been available for several years now. Satellite radars Sentinel-1 collect the data using two polarization configurations, thus providing, in principle, greater potential than their predecessors for the inventory and monitoring of land cover changes and vegetation monitoring on finer scales. Change detection in SAR images is getting increased attention in recent years for the imaging characteristics of SAR, such as all-time, all-weather, and largearea. Hence, these sensors can be used to cartography and measure changes in the state of land cover or habitat quantity/quality (Nagendra et al., 2013; Niculescu et al., 2016, 2017), as well as generate categorical products (thematic maps). Such data can be used in combination with discretely collected field data (Newton et al., 2009) to test hypotheses relating to biodiversity change (e.g., on species - habitat relationships). The use of multi-temporal radar satellite remote-sensing has been mainly focused on the assessment of changes in both habitat quantity and quality within categorized land-use classes. As well as remote-sensing, quantitative spatial-analytical approaches to conservation have arisen from applied landscape ecology studies with these aimed at understanding the relationships between spatial patterns of land-cover change and ecological, biophysical and/or socio-economic processes (Mairota et al., 2015).

For image classification, one possible way to address the categorization task is to use deep learning algorithms, for instance a convolutional neural network $(\mathrm{CNN})$. Deep learning is an important branch of machine learning, and it tries to learn abstract concepts by simulating the cognitive mechanism of human brain and explore the latent pattern by establishing deep architecture (Arel et al., 2010). When data is fed into a deep network, the features can be learned layer by layer, and the output of one layer can be taken as the input of the next layer (Bengio et al., 2013). CNN is inspired by the receptive fields in neural cortex, and it is a multilayer neural network suitable for processing 2-D data such as videos and images. Deep-learning- 
based methods, which achieve many improvements in many research fields, have been widely applied in natural images classification, object recognition, natural language, and text processing. The remote sensing community has also started to incorporate deep CNNs to image classification tasks. However, the majority of research using deep CNNs in the remote sensing community has been focusing on high-resolution images.

Classification of these high-resolution images is similar to object recognition in computer vision, and remarkable improvements achieved by deep networks in object recognition have also been shown in these applications (Sharma et al., 2017). Due to their remarkable performance, these methods are used to analyze HRRS images, and have achieved more impressive results than the traditional shallow methods for scene classification (Castelluccio et al.; Hu et al., 2015; Zhang et al., 2016; Zhao and Du, 2016; Luo et al., 2017; Wang et al., 2017; Cheng et al., 2016). Gong et al. (2016) used deep learning to achieve change detection for SAR images. They select samples based on a pre-classification without using difference image. Deep learning was then used to learn highorder features and classify the SAR images. Deep learning has shown promising performance in classification problems and it achieves accurate results.

In the second part of this work the results of the classification Deep Learning are compared with outcomes of classification of Random Forest. A random forest (RF) classifier is an ensemble classifier that produces multiple decision trees, using a randomly selected subset of training samples and variables. This classifier has become popular within the remote sensing community due to the accuracy of its classifications. The RF classifier yields reliable classifications using predictions derived from an ensemble of decision trees (Breiman, 2001). Furthermore, this classifier can be successfully used to select and rank those variables with the greatest ability to discriminate between the target classes (Belgiu and Dragut, 2016). The RF classifier is an ensemble classifier that uses a set of Classification and Regression Tree (CART) to make a prediction (Breiman, 2001). The trees are created by drawing a subset of training samples through replacement (a bagging approach). This means that the same sample can be selected several times, while others may not be selected at all. About two thirds of the samples (referred to as in-bag samples) are used to train the trees with the remaining one third (referred to as out-of-the bag samples) are used in an internal crossvalidation technique for estimating how well the resulting RF model performs (Breiman, 2001). The RF classifier has been used to map Land cover classes (Colditz, 2015; Haas and Ban, 2014; Stefanski et al., 2013; Tsutsumida and Comber, 2015), to map boreal forest habitats (Räsänen et al., 2013), to map biomass using (Frazier et al., 2014), to identify tree (Wang et al., 2015), and to map tree canopy cover and biomass using unitemporal and multi-temporal Landsat 8 imagery (Karlson et al., 2015), to map the ecosystems remediated in Danube delta using multi-temporal Sentinel-1 and Sentinel-2 images (Niculescu et al., 2017).

\subsection{Vegetation of the Study area}

The Danube Delta, Romania's youngest landmass, is a fluvialmaritime floodplain on two floristic provinces, the lower Danube (ponto-sarmatic) and the Black Sea (euxinic) (Borza, 1960; Ciocârlan, 1994). The diverse geomorphology, soils, and hydrological conditions favour the proliferation of a large number of aquatic, semi-desert, and saline habitats. At the international level, almost all habitats are considered very important. By the same token, each habitat is part of a unique nature conservation network. The flora in the Danube Delta Biosphere Reserve (both Romanian and Ukrainian sectors) is specific for a steppe bioregion with a temperate climate, featuring almost 1,400 species of vascular plants (Hanganu et al., 2002) of which five species (1 subspecies) are endemic $(0.51 \%$ of the total number).

The delta's marine zone is geo-morphologically characterized by the presence of parallel sandy beach barriers separated by shallow depressions. Most beach barriers are narrow and low, measuring several tens to a few hundreds of meters wide and lying $1.0-1.5$ meters above sea level. The depressions between them are relatively wide; many of them are hundreds to several thousand of meters across. Three complexes occur in which the barriers are wider and the depressions narrower: the Sărăturile complex, the Caraorman complex, the Letea complex. Geomorphologically, the marine zone consists of narrow beach barriers with very wide depressions in between. The crests of major beach barriers (for instance, Buhaz, Palade, and Crasnicol) are 1-1.5 meters above sea level. They are out of the reach of flooding. They often even too high to be influenced by the saline groundwater. The terrain consists of shifting sands and pastureland featuring Bermuda grass (Cynodon dactylon), silky wind grass (Apera spica-venti ssp. maritima), corn brome (Bromus squarrosus) and roundhead bulrush (Holoschoenus vulgaris). The beach barrier soil, at intermediate elevation, is still moderately saline.

The vegetation on these saline calcaric arenosols consists of a moderately salt-tolerant pasture of alkali grass (Puccinellia convoluta), P. distans, Apera spica-venti ssp. maritima and redtop (Agrostis gigantea ssp. pontica). Further on, past this Puccinellia convoluta zone, the increasing influence of fresh water flooding (up to three months a year) decreases the saline content. Agrostis gigantea ssp. pontica, rush (Juncus gerardi) and reed (Phragmites australis) are characteristic of this dynamic habitat, with alternating fresh water flooding and moderate saline levels. The next, lower zone, which floods for three to six months per year, is covered by sedge marshes, with reed mace and some reeds. The depressions themselves, with a flooding period of over six months per year, are covered by reed marshes with some sedge, growing in peat beds. Some of the younger depressions will still be in the process of being filled up with reed peat. Small lakes occur in their centre. These lakes are the last remnants of the lagoon. Reeds dominate the plaur in these small lakes. High saline levels means glasswort (Salicornia patula) and seepweed (Suaeda prostrata) are rare in 
this area, only present in a few isolated depressions within beach barriers not flooded by fresh water.

\subsection{Data set}

We used the following satellite images in this study: 20 Sentinel-1 images acquired between 9.10.2014 and 01.04.2016 (table 1). The Sentinel-1 data were acquired in a time series that covered the entire growth season of 2015 and part of 2016. This enabled us to determine the influence of the time dimension and of the polarimetric dimension ( $\mathrm{VV}$ and $\mathrm{VH}$ polarization are available) on the characterization and classification of the vegetation in coastal area of Danube delta.

\begin{tabular}{|c|c|c|}
\hline Date & $\begin{array}{c}\text { Incidence } \\
\text { angle }\end{array}$ & Orbit \\
\hline $09-10-2014$ & 38.055 & Ascending \\
\hline $02-11-2014$ & 38.786 & Descending \\
\hline $26-11-2014$ & 38.653 & Descending \\
\hline $13-01-2015$ & 39.215 & Ascending \\
\hline $26-03-2015$ & 39.856 & Ascending \\
\hline $07-04-2015$ & 38.569 & Ascending \\
\hline $01-05-2015$ & 38.421 & Descending \\
\hline $13-05-2015$ & 39.654 & Descending \\
\hline $30-06-2015$ & 39.478 & Ascending \\
\hline $05-08-2015$ & 38.665 & Descending \\
\hline $17-08-2015$ & 37.789 & Descending \\
\hline $29-08-2015$ & 38.669 & Ascending \\
\hline $10-09-2015$ & 39.285 & Descending \\
\hline $22-09-2015$ & 39.456 & Ascending \\
\hline $09-11-2015$ & 38.721 & Descending \\
\hline $03-12-2015$ & 38.451 & Ascending \\
\hline $27-12-2015$ & 39.885 & Ascending \\
\hline $20-01-2016$ & 38.411 & Descending \\
\hline $13-02-2016$ & 39.662 & Ascending \\
\hline $01-04-2016$ & 39.453 & Ascending \\
\hline
\end{tabular}

Table 1: Sentinel-1 imagery used in this study

Since it was first launched in April 2014, the Sentinel-1 satellite has allowed specialists to monitor the earth's surface day and night regardless of weather conditions and has transmitted highresolution space images free of charge. The Sentinel 1 SAR mission is part of the Copernicus Programme - European Earth Observation Programme, which was previously called GMES (Global Monitoring for Environment and Security), of the European Space Agency. Placed on an orbit at an altitude of $693 \mathrm{~km}$, Sentinel-1 operates in four data collection modes: the StripMap (SM) mode, the Interferometric Wide swath (IW) mode, the Extra-Wide swath (EW) mode and the Wave (WV) mode. Each mode provides different products with respect to spatial resolution and imaging swath. Sentinel-1 images are captured in $\mathrm{C}$ band $(5.5 \mathrm{~cm})$, and they may exhibit simple $\mathrm{HH}$ or VV polarization or double $\mathrm{HH}+\mathrm{HV}$ or $\mathrm{VH}+\mathrm{VV}$ polarization. The data used in our research were collected in the IW mode. This mode includes three sub-swaths, namely IW1, IW2 and IW3, which correspond to cyclical antenna deviations. This mode provides GRD (Ground Range Multilook Detected) and SLC (Single Look Complex) images made up of three IW (MDA, 2011). The GRD images are Multilook images (five looks for the IW mode) with less speckle noise and coarser space resolution. Although the SLC products have finer resolution, it is difficult to use them directly due to the phase information, which seems useless as it prevents extraction of additional information in certain cases.

GRD image calibration is vital for viewing the maximum amount of information on an image. In our research, the or 0 value is extracted using Calibration Tools of the OrfeoToolbox software, which provides us with the backscattering coefficient of the area. These values depend on the targets illuminated by the beam, on ground roughness and moisture and, in the end, on the vegetation density.

\section{METHODOLOGY}

\subsection{Deep Learning}

Recently, recurrent neural network (RNN) approaches have demonstrated their quality in the remote sensing field to produce land use mapping using time series of optical images (Ienco et al., 2017) and recognize vegetation cover status using Sentinel-1 radar time series (Minh et al., 2018). Motivated by these recent works, we decided evaluate the quality of RNN for our task. We chose to use the GRU unit (Gated Recurrent Unit) introduced by (Cho et al., 2014), coupled with an attention mechanism (Britz et al., 2017). Attention mechanisms are widely used in automatic signal processing (language or 1D signal) and they allow to combine together the information extracted by the GRU model at the different timestamps. The input of a GRU unit is a sequence $\left(\mathrm{x}_{\mathrm{t} 1}, \ldots, \mathrm{x}_{\mathrm{tN}}\right)$ where a generic element $\mathrm{x}_{\mathrm{ti}}$ is a multidimensional vector and it refers to the corresponding date in the time series. In our case, $\mathrm{x}_{\mathrm{ti}}$ corresponds to a vector with two components (polarizaion) $\mathrm{VV}$ and $\mathrm{VH}$ for a particular date.

The output returned by the GRU model is a sequence of feature vectors learned for each date: $\left(h_{t 1}, \ldots, h_{t N}\right)$ where each $h_{t i}$ has the same dimension d. Their matrix representation $H \in \mathrm{R}^{\mathrm{Nxd}}$ is obtained vertically stacking the set of vectors. The attention mechanism allows to combine together these different vectors $\mathrm{h}_{\mathrm{ti}}$, to combine the information returned by the GRU unit at each of the different timestamps. The attention formulation we used, considering a vector sequence of learned features $\left(h_{t 1}, \ldots\right.$, $\mathrm{h}_{\mathrm{tN}}$ ), is the following one:

$$
\begin{gathered}
\mathrm{va}_{\mathrm{a}}=\tanh \left(\mathrm{H} * \mathrm{~W}_{\mathrm{a}}+\mathrm{b}_{\mathrm{a}}\right) \\
\lambda=\operatorname{SoftMax}\left(\mathrm{v}_{\mathrm{a}} * \mathrm{u}_{\mathrm{a}}\right) \\
\text { rnn_feat }=\Sigma \lambda_{\mathrm{i}} * \mathrm{~h}_{\mathrm{ti}}
\end{gathered}
$$

Matrix $\mathrm{W}_{\mathrm{a}} \in \mathrm{R}^{\mathrm{d}, \mathrm{d}}$ and vectors $\mathrm{b}_{\mathrm{a}}, \mathrm{u}_{\mathrm{a}} \in \mathrm{R}^{\mathrm{d}}$ are parameters learned during the process. These parameters allow to combine the vectors contained in matrix $\mathrm{H}$. The purpose of this 
procedure is to learn a set of weights $\left(\lambda_{\mathrm{t} 1}, \ldots, \lambda_{\mathrm{tN}}\right)$ that allows to weight the contribution of each timestamp $\left(\mathrm{h}_{\mathrm{ti}}\right)$ through a linear combination. The $\operatorname{SoftMax}(\cdot)$ (Ienco et al., 2017) function is used to normalize weights $\lambda$ so that their sum is equal to 1 . The RNN model learns a new representation of the input sequences but it does not make any prediction by itself. To this end, a SoftMax layer is used again on top of the learned features rnn_feat to perform the final multi-class prediction.

The Deep Learning method has been implemented in Python through the Tensorflow library.

\subsection{Random Forest}

Second step, we performed synthetic Random Forest classifications for all the Sentinel-1 radar. Random Forest is an ensemble learning technique and builds upon multiple decision trees. Each decision tree is built using a subset of the original training data and is evaluated based on the remaining training features. New objects are classified as the class that is predicted by the most trees (figure 1). Each decision tree is independently produced without any pruning an each node is split using a user-defined number of features (Mtry), selected at random. By growing the forest up to a user-defined number of trees (Ntree), the algorithm creates trees that have high variance and low bias (Breiman, 2001). As mentioned above, two parameters need to be set in order to produce the forest trees: the number of decision trees to be generated (Ntree) and the number of variables to be selected and tested for the best split when growing the trees (Mtry). Theoretical and empirical research has highlighted that classification accuracy is less sensitive to Ntree than to the Mtry parameter (Ghosh et al., 2014; Kulkarni and Sinha, 2014).

According to Rodriguez-Galiano et al., 2012, the classifier has three main advantages for land cover classifications from remote-sensing images: (i) it reaches higher accuracies than other machine-learning classifiers; (ii) it has the ability to measure the importance level of the input images; (iii) it makes no assumptions about the distributions of the input images (cited by Hütt et al., 2016).

We used the following parameters for the Random Forest algorithm: 200 trees, maximum depth of the tree 25 and minimum number of samples in each node 25 .

Concerning the Random Forest Classifier, we use the public available implementation supplied by the Scikit-Learn python machine learning library.

\section{Random Forest Simplified}

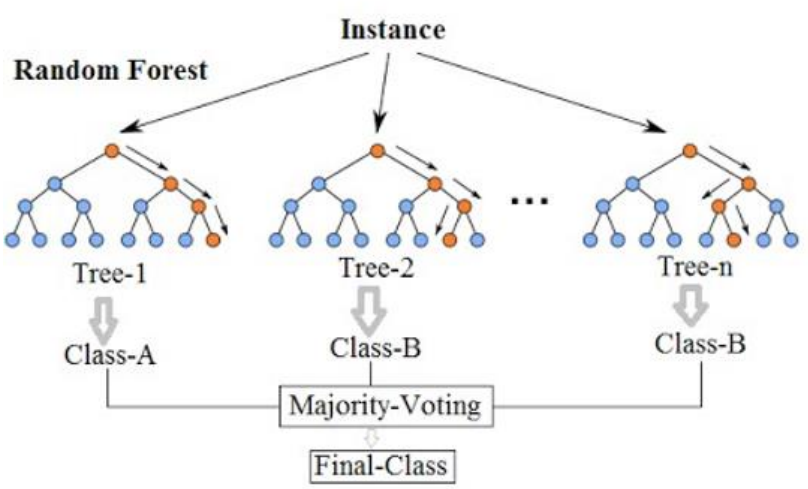

Figure 1: Performance of the Random Forest algorithm

\section{RESULTS}

The results of this study relate to application a two algorithms of remote sensing classification (Deep learning and Random Forest) from satellite sensors Sentinel-1 time series. The results of classification supervised of the two algorithms of data have been compared. This algorithms are used to improve the accuracy of recognition and mapping of major vegetation classes in the in the Danube Delta namely in its coastal zones.

The vegetation types were labeled according to 10 classes (figures classifications, figure 2 and figure 3 ). These classifications allowed us to distinguish several classes of reeds in the 'large marsh vegetation' class (reed vegetation on salinized soils, pure reed vegetation, and reed vegetation on open plaur (floating vegetation called plaur (floating reed bed) is an association of reeds and other wetland plants that grow on a one-meter thick cover made up of roots, soil and various organic materials) and two classes of reed vegetation on compact plaur (one class with cut reeds).

The results show very good classification performance for the two algorithms: $96,2 \%$ mean accuracy for deep learning and 94,3\% for Random Forest. The mapping accuracies were summarized using confusion matrices (figure 2 and 3 ) and statistics including user, producer and overall accuracy and Cohen's K.

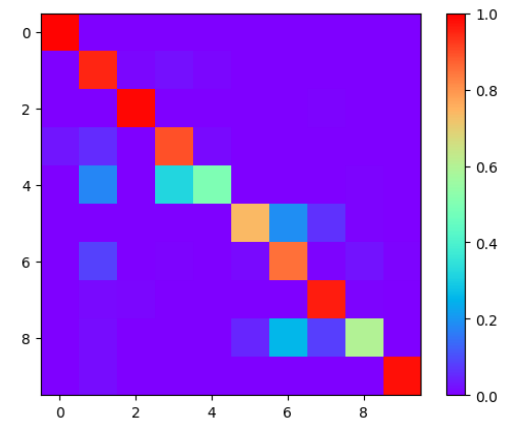


Figure 2: Matrix Confusion of Deep Learning Classification

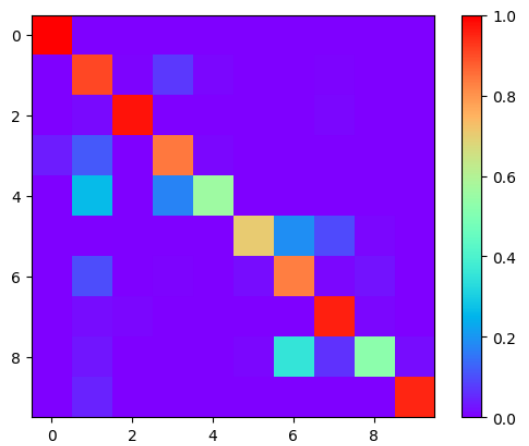

Figure 3: Matrix Confusion of Random Forest Classification

Classification accuracy was assessed using global and Kappa indices. Very good Kappa indices were obtained; for Random Forest, the Kappa index was 0.86, and for the Deep Learning the Kappa index was 0.96 . The results are obtained by a data splits in which $10 \%$ of the data are used to learn the model and $90 \%$ are used to test it. The results are expressed in percentages with respect to the reference labels, and therefore, values in the diagonal represent Producers Accuracy. The confusion matrix of the classification resulting from the Sentinel-1 time series processing reveals very good Producers Accuracy values; most classes show values ranging from $90.01 \%$ to $99.72 \%$. The most substantial confusions concern the 'pure reed vegetation class', with a Producers Accuracy of $90.01 \%$. This class is mixed with the 'reed on compact plaur' class $(4.21 \%)$ and with the 'reed vegetation on salinized soils' class (1.26\%) (figure 4 and 5). Radar data provide information especially on plant physiognomies. This analysis supplies information on polarimetric data in relation to the geometric characteristics of the physiognomies of the plants growing in delta and enables us to draw conclusions about ways to distinguish among the various plant physiognomies.

Finally, the F-measure was calculated (table 2). The Fmeasure is the harmonic mean of the precision and recall (this indicator gives the proportion of pixels well classified for each class). We can first note that these baseline scores are quite high, demonstrating the relevance of the temporal dimension for land-cover classification. In addition, note that due to the significant number of classes in the reference map, the Kappa scores are quite high and a small increase of the score can correspond to a major difference in the classification.

\begin{tabular}{|l|l|c|}
\hline Class & $\begin{array}{c}\text { Deep } \\
\text { Learning } \\
\text { F-measure }\end{array}$ & $\begin{array}{c}\text { Random } \\
\text { Forest } \\
\text { F-measure }\end{array}$ \\
\hline $\mathbf{1}$ & 0.99 & 0.99 \\
\hline $\mathbf{2}$ & 0.97 & 0.94 \\
\hline $\mathbf{3}$ & 0.61 & 0.76 \\
\hline $\mathbf{4}$ & 0.32 & 0.15 \\
\hline $\mathbf{5}$ & 0.22 & 0.27 \\
\hline $\mathbf{6}$ & 0.69 & 0.74 \\
\hline $\mathbf{7}$ & 0.73 & 0.67 \\
\hline $\mathbf{8}$ & 0.93 & 0.92 \\
\hline $\mathbf{9}$ & 0.73 & 0.67 \\
\hline $\mathbf{1 0}$ & 0.97 & 0.96 \\
\hline F-mesure & $\mathbf{0 . 9 6}$ & $\mathbf{0 . 9 5}$ \\
\hline
\end{tabular}

Table 2: F-measure of Deep Learning and Random Forest

The outcomes of F-measure for the two algorithms we show very good results for all classes of reed: 'reed vegetation on salinized soils' (0.69 for Deep Learning and 0.74 for Random Forest), 'pure reed vegetation' ( 0.73 for DP and 0.67 for RF), 'reed on open plaur' (0.93 and 0.92), 'reed on compact plaur' (0.73 and 0.67) and 'reed on compact plaur' (cut reed) (0.97 and 0.96). The class 'Dunes' and 'Dunes vegetation' present values a mediocre F-measure for the two algorithms (figure 6).

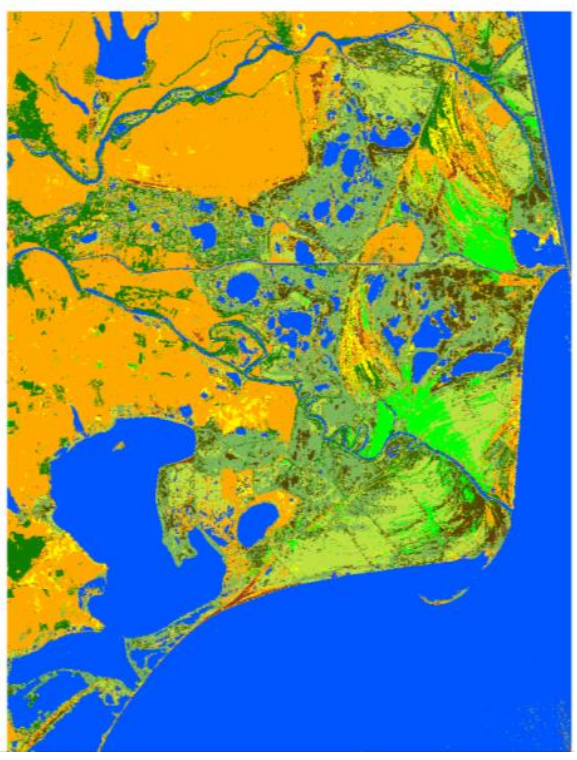

Figure 4: Deep Learning classification 


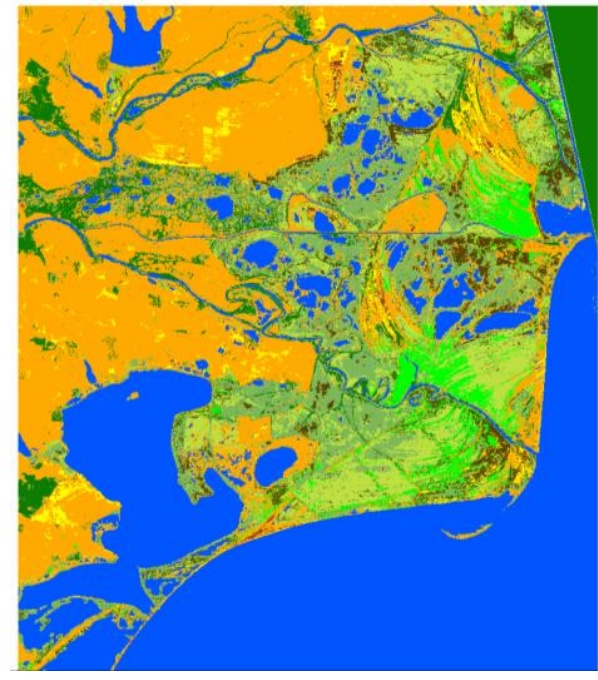

Figure 5: Random Forest Classification

\section{Permanent water Agricol polder \\ Forest \\ Dunes (sand) \\ Dunes vegetation \\ Reed vegetation on salinised soils \\ Pure reed vegetation \\ Reed on open plaur \\ Reed on compact plaur \\ Reed on compact plaur (cut reed)}

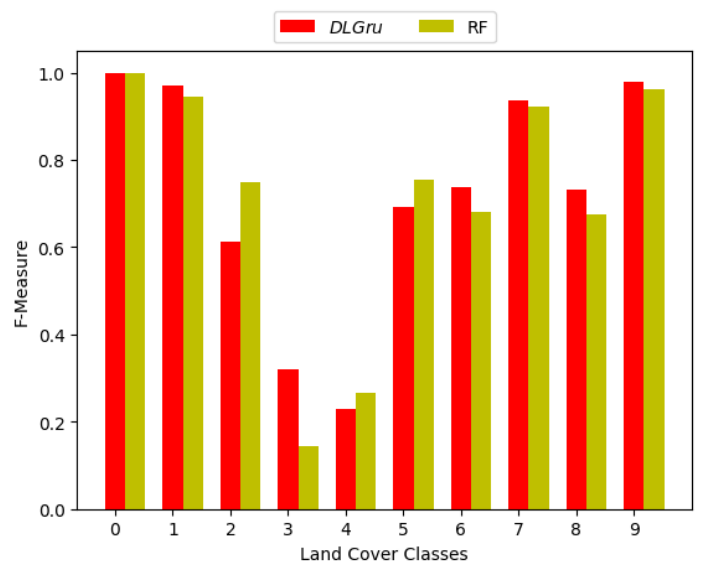

Figure 6: Performance of F-measure for the time series classification by class for Deep learning algorithm and Random Forest

Even if the scores of F-measure for the class 'Dunes vegetation' (table 2) remain modest with the two algorithms, the mapping of this class is better with the Deep Learning algorithm (figure $7, \mathrm{~B})$. The mapping of this class with this algorithm shows us the areas where the ground water is not deep and where the vegetation is installed thanks to this moisture. Ground water flows easily, and in large quantities, through the highly permeable subsoil, carried along by layers high in shell fragment. The dunes are covered with open steppe vegetation such as Carex colchica, sea grape (Ephedra distachya), wild rye (Secale silvestre), Volga wild rye (Elymus giganteus) and Festuca beckeri.

In the mapping of vegetation classes, the class 'Forest' is better mapped with the algorithm Deep Learning figure 7, D). The figure presented here represents the forest of Letea (figure 7, C and D). These natural forest ecosystems grow on the Danube Delta Biosphere Reserve's oldest fluvio-maritime sand dunes. The habitat of the forests on these dunes is very diverse. They range from floodplain river levee forests to saline forests and inland forest white poplar (Populus alba), marsh ash (Fraxinus pallisiae), marsh apple (Malus dasyphylla) and wild apple (M. sylvestris), wild pear (Pyrus pyraster) and white oak (Quercus pedunculiflora).

The third example taken in this analysis on the mapping of plant formations concerns the class 'Reed vegetation on salinized soil' (figure 7, E and F). For this class the mapping is better with the algorithm Deep learning (figure 7, F). Reeds, covering more than 220,000 hectares, are by far the Danube Delta's dominant species. The primary plant community is Scirpo-Phragmitetum. It can be found on neutral $\mathrm{pH}$, hydromorphous, and organic soils with low to moderate saline levels. The dominant species is Phragmites australis, usually accompanied by hydrophilous species as Typha angustifolia, Schoenoplectus lacustris, and Sparganium erectum, Thelypteris palustris. The various community-types are distinguishable.

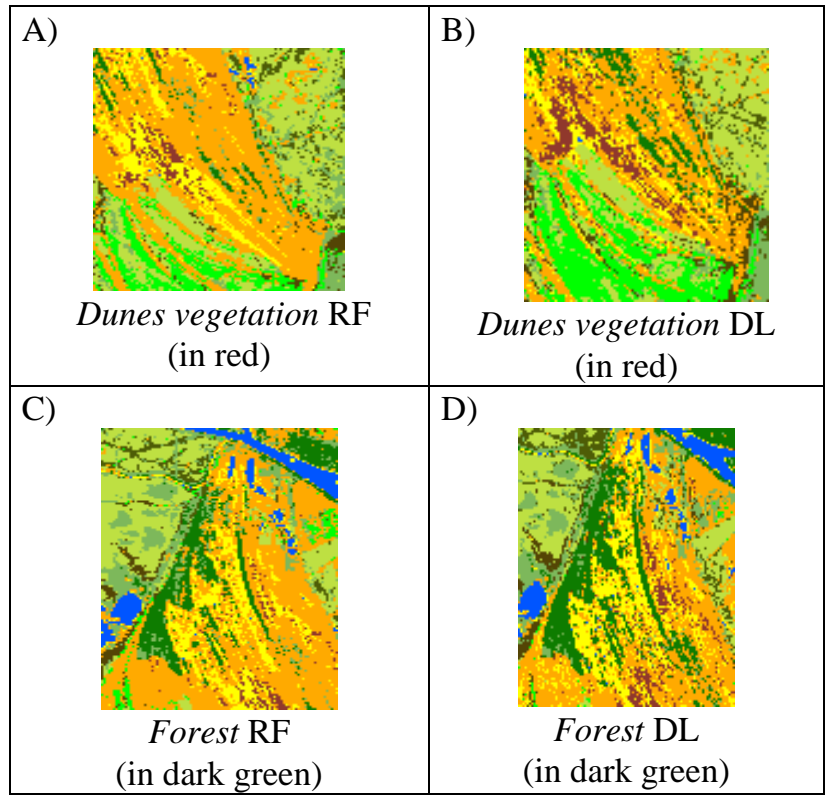




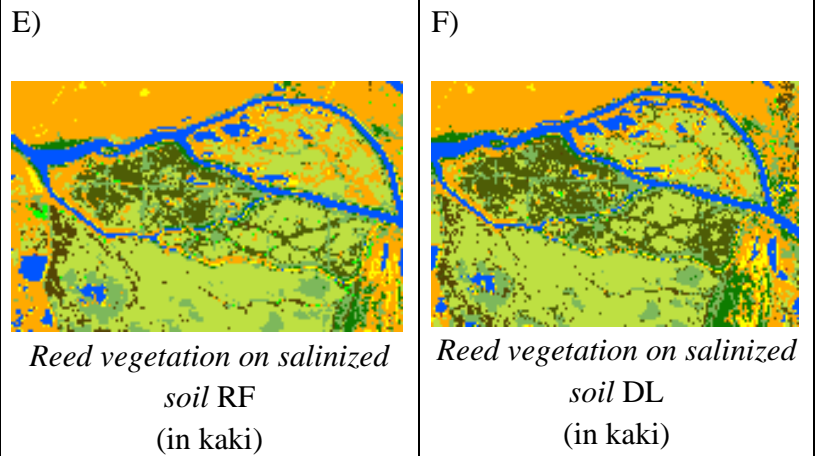

Figure 7: Comparisons of the mapping of the three classes of vegetation in the classifications of RF and DL

\subsection{Discussion and Conclusion}

Classification of Sentinel-1 series imagery (20 images) using two different machine learning (Deep Learning and Random Forest) algorithms were implemented, evaluated, and compared. The methods used have shown a real interest for the characterization of the major vegetation types and for the precise delimitation of several types of vegetation formations from the Sentinel-1 time series images. The classification procedures produced from the 20 images are reproducible which allows their implementation on vast territories.

Both methods are only based on a few input parameters and provide accurate classification results. Thus, Random Forests and Deep Learning can be regarded as a simple yet accurate approach. The classifications accuracies were increased by introducing the spatial features to original polarimetric coherency feature. Especially, multi-feature combination shows potential capability to distinguish the classes with similar polarimetric responses but different textual and spatial features, such as the all classes of reed: reed vegetation on salinized soil, pure reed vegetation, reed on open plaur, reed on compact plaur and reed on compact plaur (cut reed). Higher classification accuracies can always be obtained by Deep Learning technique that smartly combines the temporal polarimetric features. The increasing size of training samples can effectively improve the classification accuracy. Deep Learning outperforms Random Forest for all the experiments in terms of accuracy, its efficiency is lower than Random Forest because it is highly affected by feature dimensionality. The main advantage of using GRU over Random Forest is that it enables to build a hierarchy of local and sparse features derived from spectral and temporal profiles while Random Forest build a global transformation of features.

Both algorithms are robust and they can be used for remotely sensed data vegetation classification. Performance of random forests is on par with other machine learning algorithms (such as Deep Learning) but it is much easier to use and more forgiving. On the other hand, compared to random forest, Deep Learning does not need extraction and selection of hand-crafted features. Such advantage, together with its success in the signal processing field has motivated researchers in the remote sensing community to investigate its usefulness for remote sensing image analysis.

\section{REFERENCES}

Arel, I., Rose, D.C., Karnowski, T.P., 2010. Deep Machine Learning - A New Frontier in Artificial Intelligence Research [Research Frontier]. IEEE Computational Intelligence Magazine 5, 13-18.

Belgiu, M., Drăguţ, L., 2016. Random forest in remote sensing: A review of applications and future directions. ISPRS Journal of Photogrammetry and Remote Sensing 114, 24-31.

Breiman, L., 2001. Random Forests. Machine Learning 45, 532.

Britz D., M. Y. Guan, and M. Luong, "Efficient attention using a fixed- size memory representation," in EMNLP, 2017, 392400

Castelluccio, M., Poggi, G., Sansone, C., Verdoliva, L. Land Use Classification in Remote Sensing Images by Convolutional Neural Networks <http://arxiv.org/abs/1508.00092.

Cheng, G., Zhou, P., Han, J., 2016. Learning rotation-invariant convolutional neural networks for object detection in VHR optical remote sensing images. IEEE Trans. Geosci. Rem. Sens. 54 (12), 7405-7415.

Cho K., B. van Merrienboer, C, Gulcehre, D. Bahdanau, F. Bougares, H. Schwenk, and Y. Bengio, "Learning phrase representations using RNN encoder-decoder for statistical machine translation," in EMNLP, 2014, pp. 1724-1734.

Colditz, R.R., 2015. An Evaluation of Different Training Sample Allocation Schemes for Discrete and Continuous Land Cover Classification Using Decision Tree-Based Algorithms. Remote Sensing 7, 9655-9681.

Ghosh, A., Sharma, R., Joshi, P.K., 2014. Random forest classification of urban landscape using Landsat archive and ancillary data: Combining seasonal maps with decision level fusion. Applied Geography 48, 31-41.

Gong, M., Yang, H., Zhang, P., 2017. Feature learning and change feature classification based on deep learning for ternary change detection in SAR images. ISPRS Journal of Photogrammetry and Remote Sensing 129, 212-225.

Haas, J., Ban, Y., 2014. Urban growth and environmental impacts in Jing-Jin-Ji, the Yangtze, River Delta and the Pearl River Delta. Int. J. Appl. Earth Obs. Geoinf. 30, 42-55.

Hanganu J., Dubyna D., Zhmud E., Grigoraş I., Menke U., Drost H., Ştefan N. and. Sărbu I, 2002, Vegetation of the 
Biosphere Reserve Danube Delta - with Transboundary Vegetation Map on a 1:150,000 scale", RIZA rapport, Lelystad,

He, K.S., Rocchini, D., Neteler, M., Nagendra, H., 2011. Benefits of hyperspectral remotesensing for tracking plant invasions. Divers. Distrib. 17, 381-392.

Hu, F., Xia, G.-S., Hu, J., Zhang, L., 2015. Transferring deep convolutional neural networks for the scene classification of high-resolution remote sensing imagery. Rem. Sens. 7 (11), 14680-14707.

Hütt, C., Koppe, W., Miao, Y., Bareth, G., 2016. Best Accuracy Land Use/Land Cover (LULC) Classification to Derive Crop Types Using Multitemporal, Multisensor, and MultiPolarization SAR Satellite Images. Remote Sensing 8.

Ienco D., R. Gaetano, C. Dupaquier, and P. Maurel, 2017, Land cover classification via multitemporal spatial data by deep recurrent neural networks, IEEE GRSL, vol. 14, no. 10, 16851689.

Karlson, M., Ostwald, M., Reese, H., Sanou, J., Tankoano, B., Mattsson, E., 2015. Mapping tree canopy cover and aboveground biomass in Sudano-Sahelian woodlands using landsat 8 and random forest. Remote Sens. 7, 10017.

Kulkarni, V.Y., Sinha, P.K., 2014. Effective Learning and Classification using Random Forest Algorithm, International Journal of Engineering and Innovative Technology (IJEIT) Volume 3, Issue 11.

Luo, X., Wang, M., Dai, G., Chen, X., 2017. A novel technique to compute the revisit time of satellites and its application in remote sensing satellite optimization design. Int. J. Aerosp. Eng. 2017.

Mairota, P., Cafarelli, B., Didham, R.K., Lovergine, F.P., Lucas, R.M., Nagendra, H., Rocchini, D., Tarantino, C., 2015. Challenges and opportunities in harnessing satellite remotesensing for biodiversity monitoring. Ecological Informatics 30, 207-214.

Minh D. H. T., D. Ienco, R. Gaetano, N. Lalande, E. Ndikumana, F. Osman, and P. Maurel, "Deep recurrent neural networks for winter vegetation quality mapping via multitemporal sar sentinel-1," IEEE GRSL, vol. Preprint, 2018.

Nagendra, H., Lucas, R.M., Honrado, J.P., Jongman, R.H.G., Tarantino, C., Adamo, M., Mairota, P., 2013. Remote-sensing for conservation monitoring: assessing protected areas, habitat extent, habitat condition, species diversity and threats. Ecol. Indic. 33, 45-59.

Newton, A.C., Hill, R.A., Echeverría, C., Golicher, D., Benayas, J.M.R., Cayuela, L., Hinsley, S.A., 2009. Remotesensing and the future of landscape ecology. Prog. Phys. Geogr.
Niculescu S., Lardeux C., Grigoras I., Hanganu J., David L., 2016, Synergy between LiDAR, RADARSAT-2 and SPOT-5 images for the detection and mapping of wetland vegetation in the Danube Delta, IEEE Journal of Selected Topics in Applied Earth Observations and Remote Sensing, vol. 9, no 8, 36513666.

Niculescu S., Lardeux C., Hanganu J., 2017, Alteration and Remediation of Coastal Wetland Ecosystems in the Danube Delta. A Remote-Sensing Approach, Coastal Research Library, vol. 21, 513 .

Räsänen, A., Rusanen, A., Kuitunen, M., Lensu, A., 2013. What makes segmentation good? A case study in boreal forest habitat mapping. Int. J. Remote Sens. 34, 8603-8627.

Rodriguez-Galiano, V.F.; Ghimire, B.; Rogan, J.; Chica-Olmo, M.; Rigol-Sanchez, J.P., 2012, An assessment of the effectiveness of a random forest classifier for land-cover classification, ISPRS J. Photogramm. Remote Sens., 67, 93104.

Sharma, A., Liu, X., Yang, X., Shi, D., 2017. A patch-based convolutional neural network for remote sensing image classification. Neural Networks 95, 19-28.

Stefanski, J., Mack, B., Waske, B., 2013. Optimization of object-based image analysis with random forests for land cover mapping. IEEE J. Sel. Top. Appl. Earth Obs. Remote Sens. 6, 2492-2504.

Tsutsumida, N., Comber, A.J., 2015. Measures of spatiotemporal accuracy for time series land cover data. Int. J. Appl. Earth Obs. Geoinf. 41, 46-55.

V. Ciocârlan, 1994, Flora Deltei Dunarii, Cormophyta, Bucuresti, Editura Ceres.

Wang, H., Zhao, Y., Pu, R., Zhang, Z., 2015. Mapping Robinia pseudoacacia forest health conditions by using combined spectral, spatial, and textural information extracted from IKONOS imagery and random forest classifier. Remote Sens. 7,

Wang, L., Zhang, J., Liu, P., Choo, K.-K.R., Huang, F., 2017. Spectral-spatial multifeature-based deep learning for hyperspectral remote sensing image classification. Soft Comput. 21 (1), 213-221.

Wulder, M.A., Hall, R.J., Coops, N.C., Franklin, S.E., 2004. High spatial resolution remotely sensed data for ecosystem characterization. Bioscience 54, 511-521.

Zhang, P., Gong, M., Su, L., Liu, J., Li, Z., 2016. Change detection based on deep feature representation and mapping transformation for multi-spatial-resolution remote sensing images. ISPRS J. Photogram. Rem. Sens. 116, 24-41.

Zhao, W., Du, S., 2016. Learning multiscale and deep representations for classifying remotely sensed imagery. ISPRS J. Photogram. Rem. Sens. 113, 155-165. 